\title{
Protein kinase D1: gatekeeper of the epithelial phenotype and key regulator of cancer metastasis?
}

\author{
Peter Storz ${ }^{\star 1}$ \\ ${ }^{1}$ Department of Cancer Biology, Mayo Clinic, Jacksonville, FL 32224, USA
}

The development of epithelial cancer and subsequent metastasis goes along with loss of cellular polarity and cell-cell connections (Gandalovicova et al, 2016). Epithelial-to-mesenchymal transition (EMT) and reorganisation of the extracellular matrix by secreted matrix metalloproteinases (MMPs) are key mechanisms in the separation of tumour cells from the surrounding cells, and their dissemination to distant locations in the body (Nistico et al, 2012). Recent work by several groups suggests that protein kinase D (PKD) enzymes serve crucial functions in regulating multiple processes in maintaining the epithelial phenotype. A new article in this issue of the British Journal of Cancer (Ganju et al, 2018) has shed further light on this role.

The PKD family of serine/threonine kinases consists of three members, PKD1, PKD2, and PKD3, which phosphorylate a broad spectrum of targets and control multiple functions within cells (Fu and Rubin, 2011). Although, with respect to tumour formation, all three isoforms have been implicated in regulating cancer cell survival and proliferation, the initiation of the metastatic process requires a shift in PKD isoform expression (Durand et al, 2015). For example, in tumours of the breast, a transition from a less aggressive to a more aggressive metastatic phenotype is characterised by PKD1 gene promoter methylation and downregulation, whereas PKD3 is upregulated in its expression (Borges et al, 2013, 2015). This is because PKD1 blocks cell motility, whereas PKD3 seems to drive this event (Durand et al, 2015).

In tumour cells, PKD1 negatively regulates the motile phenotype of cells through multiple mechanisms involving the regulation of focal adhesion dynamics (Jaggi et al, 2005; Durand et al, 2016), actin reorganisation dynamics at the leading edge (Eiseler et al, 2009b; Spratley et al, 2011; Doppler et al, 2014), and filopodia formation (Doppler et al, 2013) (Figure 1). This is achieved through phosphorylation of a multitude of substrates, with a net effect of complete downregulation of processes that drive cell migration and invasion. For example, cofilin is a molecule that mediates F-actin severing, leading to the formation of free barbed ends that are needed for actin branching and leading-edge progression towards a stimulus (Bravo-Cordero et al, 2013; Mizuno, 2013). PKD1 has been shown to block cofilin at multiple levels, such that the pool of inactive, S3-phosphorylated cofilin accumulates. This is achieved by PKD1-mediated negative regulation of the phosphatase Slingshot 1L (SSH1) (Eiseler et al, 2009b), as well as phosphorylation-mediated activation of p21activated kinase 4 (PAK4), which is an upstream kinase of the cofilin kinases LIMK1/2 (Spratley et al, 2011; Doppler et al, 2014). Focal adhesion dynamics and filopodium formation are regulated by PKD1 through phosphorylation of phosphatidylinositol-4phosphate 5-kinase type-1 $\gamma$ (PIP5K1 $\gamma$ ) and vasodilator-stimulated phosphoprotein (Doppler et al, 2013; Durand et al, 2016). In addition to this, PKD1 blocks extracellular matrix degradation by decreasing the expression of MMPs (Eiseler et al, 2009a), whereas the two other PKD isoforms induce their expression (LaValle et al, 2012; Wille et al, 2014).

PKD1 also maintains the epithelial phenotype of cells by negatively regulating key molecules that control EMTs. For example, PKD1 phosphorylates Snail (SNAI1), a transcriptional repressor for $\mathrm{CDH} 1$ (encoding E-cadherin) in the nucleus of breast cancer cells (Bastea et al, 2012), leading to nuclear export (Du et al, 2010) and subsequent proteasomal degradation of Snail (Zheng et al, 2014). In addition to regulating E-cadherin at the gene level, Jaggi and colleagues demonstrated in their previous work that $\mathrm{PKD} 1$ regulates the nuclear/cytosolic shuttling of $\beta$-catenin ( $\mathrm{Du}$ et al, 2009), and therefore controls the formation of E-cadherinmediated cell-cell contacts (Jaggi et al, 2005).

In this issue of the British Journal of Cancer, Ganju et al (2018) now expand the insight into this functional aspect of PKD1mediated regulation of the epithelial phenotype. They discovered

*Correspondence: Dr P Storz; E-mail: storz.peter@mayo.edu 


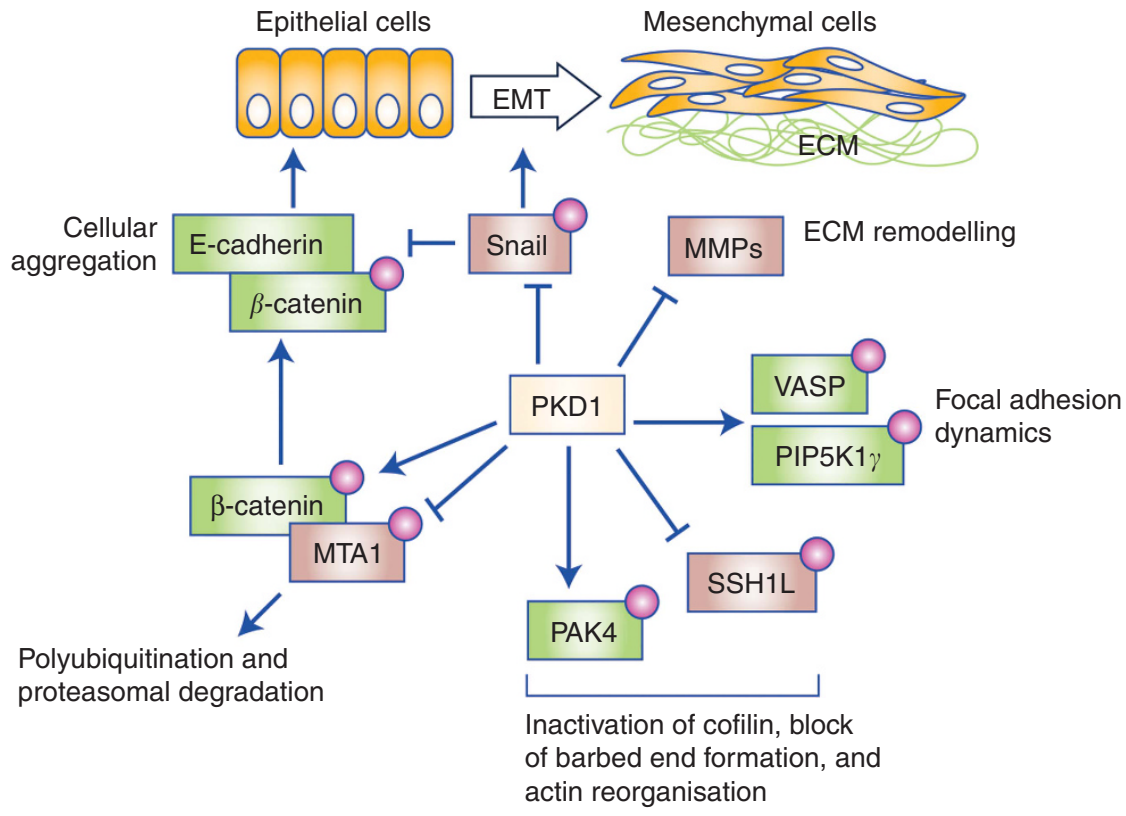

Figure 1. Roles of PKD1 in maintaining the epithelial phenotype. Shown are some of the key events that are regulated by PKD1 and that prevent EMT and decrease cell motility. PKD1 blocks actin cytoskeleton rearrangements needed for cell movement through phosphorylation of SSH1L (inactivation) and PAK4 (activation), with a net effect of inhibiting cofilin. PKD1 also affects filopodia formation and length through phosphorylation of VASP, as well as focal adhesion dynamics by targeting PIP5K1 $\gamma$ and VASP. EMT and remodelling of the extracellular matrix are regulated through phosphorylation and proteasomal degradation of Snail, and by inhibition of MMP expression. In addition to increasing E-cadherin expression through inactivation of Snail, PKD1 also regulates the formation of E-cadherin-mediated cell-cell connections through phosphorylation of MTA1 and $\beta$-catenin, leading to MTA1 proteasomal degradation and $\beta$-catenin location to the E-cadherin complexes. EMT=epithelial-tomesenchymal transition; MMPs=matrix metalloproteinases; MTA1=metastasis-associated protein 1; PKD1=protein kinase D1.

metastasis-associated protein 1 (MTA1) as a novel substrate for PKD1, and show that PKD1-mediated phosphorylation of MTA1 triggers polyubiquitination and proteasomal degradation. Moreover, they propose that this leads to the shuttling of $\mathrm{PKD} 1 / \beta$ catenin complexes to E-cadherin, with the effect of stabilising cellcell contacts. In addition to providing this mechanistic insight at a cellular level, Ganju et al also demonstrated a reverse correlation between PKD1 and MTA1 expression in samples of human prostate, colon, and breast cancers, in which PKD1 expression decreased and MTA1 expression increased, with progressed tumour grade or stage. Since PKD1 has such marked negativeregulatory effects on cell-cell aggregation, EMT, cell migration, and invasion, it is not surprising that it is epigenetically downregulated in many invasive cancers.

The present work by Ganju et al provides additional strong in vitro and in vivo evidence that PKD1 indeed is a gatekeeper of the epithelial phenotype and prevents cell migration and invasion at multiple levels; however, important questions remain. For example, with respect to the dual roles of PKD isoforms in cancer progression, it remains unclear how PKD1 can have such profound negative-regulatory effects on cell motility, whereas PKD3 seems to act in the exact opposite manner. It may be explained by differences in the regulatory elements of these kinases such as the PDZ-binding motif at the $\mathrm{C}$ terminus, which only occurs in PKD1 and PKD2, but not in PKD3 (Durand et al, 2015). Moreover, the different functions between PKD isoforms in the invasive stages of cancer also leads to the question of whether panPKD inhibitors are suitable tools for therapy. This, in part, has been answered for pancreatic, bladder, colorectal, and breast cancers (Harikumar et al, 2010; Wei et al, 2014; Borges et al, 2015; Li et al, 2017). In triple-negative and oestrogen receptor-negative invasive breast cancers, for example, which express PKD2 and PKD3, a pan inhibitor (CRT0066101) in mice has been shown to reduce primary tumour growth as well as metastasis to the lung (Borges et al, 2015); however, effects of this inhibitor on breast cancers that also express PKD1 have not yet been tested. Although, one would expect that treatment of primary tumours will be affected in their proliferation, which is regulated by all three PKDs, one could assume that inhibition of PKD1 would conversely lead to higher motility of cancer cells. However, the simultaneous inhibition of PKD2 and PKD3, which are both needed for motility and invasiveness, should prevent spreading and metastasis.

In summary, while the exact roles of PKD isoforms in many aspects of tumour initiation, progression, and metastasis are still ill-defined, the work by Ganju et al now adds important in vitro and in vivo findings, which contribute to our understanding of how PKD1 maintains the epithelial phenotype. This information is also needed in order to judge the use of PKD inhibition strategies for several human cancers, and to evaluate the patient groups that could benefit most from therapy with pan-PKD inhibitors.

\section{ACKNOWLEDGEMENTS}

The present work was supported by the NIH grants CA184527 and CA200572 from the National Cancer Institute, National Institute of Health.

\section{CONFLICT OF INTEREST}

The author declares no conflict of interest.

\section{REFERENCES}

Bastea LI, Doppler H, Balogun B, Storz P (2012) Protein kinase D1 maintains the epithelial phenotype by inducing a DNA-bound, inactive SNAI1 transcriptional repressor complex. PLoS One 7(1): e30459. 
Borges S, Doppler H, Perez EA, Andorfer CA, Sun Z, Anastasiadis PZ, Thompson E, Geiger XJ, Storz P (2013) Pharmacologic reversion of epigenetic silencing of the PRKD1 promoter blocks breast tumor cell invasion and metastasis. Breast Cancer Res 15(2): R66.

Borges S, Perez EA, Thompson EA, Radisky DC, Geiger XJ, Storz P (2015) Effective targeting of estrogen receptor-negative breast cancers with the protein kinase D inhibitor CRT0066101. Mol Cancer Ther 14(6): 1306-1316.

Bravo-Cordero JJ, Magalhaes MA, Eddy RJ, Hodgson L, Condeelis J (2013) Functions of cofilin in cell locomotion and invasion. Nat Rev Mol Cell Biol 14(7): 405-415.

Doppler H, Bastea LI, Borges S, Spratley SJ, Pearce SE, Storz P (2014) Protein kinase $\mathrm{d}$ isoforms differentially modulate cofilin-driven directed cell migration. PLoS One 9(5): e98090.

Doppler HR, Bastea LI, Lewis-Tuffin LJ, Anastasiadis PZ, Storz P (2013) Protein kinase D1-mediated phosphorylations regulate vasodilatorstimulated phosphoprotein (VASP) localization and cell migration. J Biol Chem 288(34): 24382-24393.

Du C, Jaggi M, Zhang C, Balaji KC (2009) Protein kinase D1-mediated phosphorylation and subcellular localization of beta-catenin. Cancer Res 69(3): 1117-1124.

Du C, Zhang C, Hassan S, Biswas MH, Balaji KC (2010) Protein kinase D1 suppresses epithelial-to-mesenchymal transition through phosphorylation of snail. Cancer Res 70(20): 7810-7819.

Durand N, Bastea LI, Long J, Doppler H, Ling K, Storz P (2016) Protein Kinase $\mathrm{D} 1$ regulates focal adhesion dynamics and cell adhesion through Phosphatidylinositol-4-phosphate 5-kinase type-l gamma. Sci Rep 6: 35963.

Durand N, Borges S, Storz P (2015) Functional and therapeutic significance of protein kinase D enzymes in invasive breast cancer. Cell Mol Life Sci 72(22): 4369-4382.

Eiseler T, Doppler H, Yan IK, Goodison S, Storz P (2009a) Protein kinase D1 regulates matrix metalloproteinase expression and inhibits breast cancer cell invasion. Breast Cancer Res 11(1): R13.

Eiseler T, Doppler H, Yan IK, Kitatani K, Mizuno K, Storz P (2009b) Protein kinase D1 regulates cofilin-mediated F-actin reorganization and cell motility through slingshot. Nat Cell Biol 11(5): 545-556.

Fu Y, Rubin CS (2011) Protein kinase D: coupling extracellular stimuli to the regulation of cell physiology. EMBO Rep 12(8): 785-796.

Gandalovicova A, Vomastek T, Rosel D, Brabek J (2016) Cell polarity signaling in the plasticity of cancer cell invasiveness. Oncotarget 7(18): 25022-25049.

Ganju A, Chauhan S, Hafeez B, Doxtater K, Tripathi M, Zafar N, Yallapu M, Kumar R, Jaggi M (2018) Protein kinase D1 regulates subcellular localisation and metastatic function of metastasis-associated protein $1 . \mathrm{Br} \mathrm{J}$ Cancer 118(4): 587-599.

Harikumar KB, Kunnumakkara AB, Ochi N, Tong Z, Deorukhkar A, Sung B, Kelland L, Jamieson S, Sutherland R, Raynham T, Charles M, Bagherzadeh A, Foxton C, Boakes A, Farooq M, Maru D, Diagaradjane P, Matsuo Y, Sinnett-Smith J, Gelovani J, Krishnan S, Aggarwal BB, Rozengurt E, Ireson CR, Guha S (2010) A novel small-molecule inhibitor of protein kinase D blocks pancreatic cancer growth in vitro and in vivo. Mol Cancer Ther 9(5): 1136-1146.

Jaggi M, Rao PS, Smith DJ, Wheelock MJ, Johnson KR, Hemstreet GP, Balaji KC (2005) E-cadherin phosphorylation by protein kinase D1/ protein kinase $\mathrm{C}\{\mathrm{mu}\}$ is associated with altered cellular aggregation and motility in prostate cancer. Cancer Res 65(2): 483-492.

LaValle CR, Zhang L, Xu S, Eiseman JL, Wang QJ (2012) Inducible silencing of protein kinase D3 inhibits secretion of tumor-promoting factors in prostate cancer. Mol Cancer Ther 11(7): 1389-1399.

Li QQ, Hsu I, Sanford T, Railkar R, Balaji N, Sourbier C, Vocke C, Balaji KC, Agarwal PK (2017) Protein kinase D inhibitor CRT0066101 suppresses bladder cancer growth in vitro and xenografts via blockade of the cell cycle at G2/M. Cell Mol Life Sci.

Mizuno K (2013) Signaling mechanisms and functional roles of cofilin phosphorylation and dephosphorylation. Cell Signal 25(2): 457-469.

Nistico P, Bissell MJ, Radisky DC (2012) Epithelial-mesenchymal transition: general principles and pathological relevance with special emphasis on the role of matrix metalloproteinases. Cold Spring Harb Perspect Biol 4(2): $1-10$.

Spratley SJ, Bastea LI, Doppler H, Mizuno K, Storz P (2011) Protein kinase D regulates cofilin activity through $\mathrm{p} 21$-activated kinase 4 . J Biol Chem 286(39): 34254-34261.

Wei N, Chu E, Wipf P, Schmitz JC (2014) Protein kinase d as a potential chemotherapeutic target for colorectal cancer. Mol Cancer Ther 13(5): 1130-1141.

Wille C, Kohler C, Armacki M, Jamali A, Gossele U, Pfizenmaier K, Seufferlein T, Eiseler T (2014) Protein kinase D2 induces invasion of pancreatic cancer cells by regulating matrix metalloproteinases. Mol Biol Cell 25(3): 324-336.

Zheng H, Shen M, Zha YL, Li W, Wei Y, Blanco MA, Ren G, Zhou T, Storz P, Wang HY, Kang Y (2014) PKD1 phosphorylation-dependent degradation of SNAIL by SCF-FBXO11 regulates epithelial-mesenchymal transition and metastasis. Cancer Cell 26(3): 358-373. 